\title{
Global Stability of Complex-Valued Genetic Regulatory Networks with Delays on Time Scales
}

\author{
Yajing Wang ${ }^{1}$, Zhenkun Huang ${ }^{1}$, Juan Chen $^{1}$ \\ 1 Jimei University, Xiamen 361021, China
}

\begin{abstract}
In this paper, the global exponential stability of complex-valued genetic regulatory networks with delays is investigated. Besides presenting conditions guaranteeing the existence of a unique equilibrium pattern, its global exponential stability is discussed. Some numerical examples for different time scales.

Keywords: Complex-Valued Genetic Regulatory Networks, Time Scales, Delay, Global Exponential Stability, globally exponentially stable.
\end{abstract}

\section{Introduction}

The study of complex-valued neural networks (CVNNs for short) is a fast growing area of research in recent times as is apparent from a large number of publications. In fact, complex-valued neural networks (CVNN) make it possible to solve some problems which cannot be solved with their real-valued counterparts. Stimulated by works in $[1,2]$, We consider the generalized CVNN described by the equation.

$$
\left\{\begin{array}{l}
z^{\Delta}(t)=-A z(t)+B f(\hat{z}(t-\tau))+L \\
\hat{z}^{\Delta}(t)=-P \hat{z}(t)+D z(t-\tau)
\end{array}\right.
$$

where $T$ be a time scale and $C$ be the set of complex numbers, $z: T \rightarrow C^{n}, \hat{z}: T \rightarrow C^{n}, A, B, P$ and $D$ are $n \times n$-matrices with complex entries, the activation functions are given by $f: C^{n} \rightarrow C^{n}$, and the inputs are given by $L \in C^{n}$.

\section{Essentials of time scales}

An arbitrary nonempty closed subset $T$ of the set of real numbers $R$ is called a time scale. In this paper, we only consider time scales that are unbounded above. We define the forward jump operator $\sigma: T \rightarrow T$ (and similarly the backward jump operator $\rho$ ) by $\sigma(t)=\inf \{s \in T: s>t\}$. A point $\mathrm{t} t \in T$ is called right-scattered, right-dense, left-scattered, left-dense, if $\sigma(t)>t, \sigma(t)=t, \rho(t)>t, \rho(t)=t \quad$ holds, respectively. The graininess $\mu: T \rightarrow[0]$ is defined by $\mu(t)=\sigma(t)-t$. For $f: T \rightarrow C^{n}$ and $t \in T$, we note that the real and imaginary parts of $f$ are real valued and one can use the time scales results below for the realvalued entries of $\operatorname{Re}(f)$ and $\operatorname{Im}(f)$. We say that $f: T \rightarrow R$ is delta differentiable at $t \in T$ provided there exists an $\alpha$ such that for all $\varepsilon>0$ there is a neighborhood $\mathcal{N}$ of $t$ with

$|f(\sigma(t))-f(s)-\alpha(\sigma(t)-s)| \leq \varepsilon|\sigma(t)-s|$ for all $s \in \mathcal{N}$.

It is easy to see that

$$
f^{\Delta}(t)= \begin{cases}\lim _{s \rightarrow s, f(t)-f(s)} \frac{f(s)}{t-s}, & \mu(t)=0, \\ \frac{f(\sigma(t))-f(t)}{\mu(t)}, & \mu(t)>0 .\end{cases}
$$

and

$$
(f g)^{\Delta}=f^{\Delta} g+f^{\sigma} g^{\Delta}=f g^{\Delta}+f^{\Delta} g^{\sigma},
$$

where we put $f^{\sigma}=f \circ \sigma$, and the simple useful formula

$$
f^{\sigma}=f+\mu f^{\Delta} .
$$

We say that a function $f: T \rightarrow R$ is regressive provided $1+\mu(t) f(t) \neq 0$ for all $t \in T$. The set of all regressive and rd-continuous functions is denoted by $\mathcal{R}$. The set $\mathcal{R}^{+}$of all positively regressive function consists of those $f \in \mathcal{R}$ that satisfy $1+\mu(t) f(t)>0$ for all $f \in \mathcal{R}$.

Lemma 1 Let $p, q \in \mathcal{R}$ and $t, s, r \in T$. Then

(i) $e_{0}\left(t, t_{0}\right) \equiv 1$ and $e_{p}(t, t) \equiv 1$;

(ii) $e_{p}(\sigma(t), s)=(1+\mu(t) p(t)) e_{p}(t, s)$;

(iii) if $T=R$ and $p(t) \equiv \alpha$, then $e_{p}(t, s)=e^{\alpha(t-s)}$;

(iv) if $p \in \mathcal{R}^{+}$, then $e_{p}(t, s)>0$ for all $t>s$. 
Lemma 2 Let $y \in C_{r d}$ and $p \in \mathcal{R}^{+}$. If $y$ is differentiable on $\left[t_{0}, \infty\right) \cap T$ such that

$$
y^{\Delta}(t) \leq p(t) y(t) \quad \text { for all } t \in\left[t_{0}, \infty\right) \cap T,
$$

then

$$
y(t) \leq y\left(t_{0}\right) e_{p}\left(t, t_{0}\right) \quad \text { for all } t \in\left[t_{0}, \infty\right) \cap T .
$$

\section{Existence of a unique equilibrium pattern}

Consider the space $C^{n}$ of all $n$-vectors of complex numbers and let $|z|=\sqrt{z^{*} z}$ denote the absolute value of $z \in C^{n}$, where ${ }^{*}$ indicates the conjugate transpose.

Theorem 3 Suppose $f: C^{n} \rightarrow C^{n}$ is continuous with $f(0)=0$ and there exists $L>0$ such that $\left|f\left(z_{1}\right)-f\left(z_{2}\right)\right| \leq L\left|z_{1}-z_{2}\right|$ for all $z_{1}, z_{2} \in C^{n}$. Define $\gamma:=\|I-A\|+\|B\|\left\|P^{-1} D\right\| L$.

If $P$ is invertable and $\gamma \in(0,1)$, then the system (1) possesses a unique equilibrium pattern.

Proof Clearly, the assumptions of the theorem imply $|f(z)| \leq L|z|$ for all $z \in C^{n}$. Now define the operator $F: C^{n} \rightarrow C^{n}$ by

$$
F(z)=(I-A) z+B f\left(P^{-1} D z\right)+L .
$$

For $z_{1}, z_{2} \in C^{n}$, we have

$$
\begin{gathered}
\left|F\left(z_{1}\right)-F\left(z_{2}\right)\right|=\left|(I-A)\left(z_{1}-z_{2}\right)+B\left[f\left(P^{-1} D z_{1}\right)-f\left(P^{-1} D z_{2}\right)\right]\right| \\
\leq \gamma\left|z_{1}-z_{2}\right| .
\end{gathered}
$$

Thus the mapping $F$ is a contraction on $C^{n}$. Therefore, $F$ has a unique fixed point $z$ in $C^{n}$ since $C^{n}$ is complete. Let $\hat{z}^{0}=P^{-1} D z^{0}$, then we get

$$
\left\{\begin{array}{l}
-C \hat{z}^{0}+D z^{0}=0 \\
-A z^{0}+B f\left(\hat{z}^{0}\right)+L=0 .
\end{array}\right.
$$

This unique fixed point $\left(z^{0}, \hat{z}^{0}\right)$ is the required equilibrium pattern for the system (1).

\section{Global exponential stability}

We assume that the system (1) possesses a unique equilibrium pattern $(\dot{z}, \ddot{z})^{T}$. Using the transformation $\bar{z}(t)=z(t)-\dot{z}, \overline{\bar{z}}(t)=\hat{z}(t)-\ddot{z}$ in system (1), we get

$$
\left\{\begin{array}{l}
\bar{z}^{\Delta}(t)=-A \bar{z}(t)+B g(\overline{\bar{z}}(t-\tau)) \\
\overline{\bar{z}}^{\Delta}(t)=-P \overline{\bar{z}}(t)+D \bar{z}(t-\tau)
\end{array}\right.
$$

where $g(z)=f(z+\ddot{z})-f(\ddot{z})$. Redesignating

$$
\begin{aligned}
(\bar{z}(t), \overline{\bar{z}}(t))^{T} & \text { as }(z(t), \hat{z}(t))^{T} \text { we obtain } \\
& \left\{\begin{array}{l}
z^{\Delta}(t)=-A z(t)+B g(\hat{z}(t-\tau)), \\
\hat{z}^{\Delta}(t)=-P \hat{z}(t)+D z(t-\tau) .
\end{array}\right.
\end{aligned}
$$

Clearly the stability of $(\dot{z}, \ddot{z})^{T}$ for the system (1) is equivalent to the stability of the trivial solution for the system (4). We use the following concept of global exponential stability.

Lemma 6 If $(z, \hat{z})^{T}$ is a solution of (4), then $w=z^{*} z+\hat{z}^{*} \hat{z}$ and $w_{1}=z^{*} z, w_{2}=\hat{z}^{*} \hat{z}$ satisfies

$$
\begin{gathered}
w^{\Delta}=z^{*}\left(-A^{*}-A+\mu A^{*} A\right) z+\mu g^{*}(\hat{z}(t-\tau)) B^{*} B g(\hat{z}(t-\tau)) \\
+g^{*}(\hat{z}(t-\tau)) B^{*}(I-\mu A) z+z^{*}(I-\mu A)^{*} B g(\hat{z}(t-\tau)) \\
+\hat{z}^{*}\left(-P^{*}-P+\mu P^{*} P\right) \hat{z}+\mu z^{*}(t-\tau) D^{*} D z(t-\tau) \\
+\hat{z}^{*}(I-\mu P)^{*} D z(t-\tau)+z^{*}(t-\tau) D^{*}(I-\mu P) \hat{z} .
\end{gathered}
$$

Proof We use the product rule (2) and the simple useful formula (3) to calculate

$$
\begin{aligned}
& w^{\Delta}=\left(-z^{*} A^{*}+g^{*}(\hat{z}(t-\tau)) B^{*}\right) z+z^{*}(-A z+B g(\hat{z}(t-\tau))) \\
&+\mu\left(-z^{*} A^{*}+g^{*}(\hat{z}(t-\tau)) B^{*}\right)(-A z+B g(\hat{z}(t-\tau))) \\
&+\left(-\hat{z}^{*} P^{*}+z^{*}(t-\tau) D^{*}\right) \hat{z}+\hat{z}^{*}(-P \hat{z}+D z(t-\tau)) \\
&+\mu\left(-z^{*} P^{*}+z^{*}(t-\tau) D^{*}\right)(-P \hat{z}+D z(t-\tau)) \\
&=z^{*}(\left.-A^{*}-A+\mu A^{*} A\right) z+\mu g^{*}(\hat{z}(t-\tau)) B^{*} B g(\hat{z}(t-\tau)) \\
&+g^{*}(\hat{z}(t-\tau)) B^{*}(I-\mu A) z+z^{*}(I-\mu A)^{*} B g(\hat{z}(t-\tau)) \\
&+\hat{z}^{*}\left(-P^{*}-P+\mu P^{*} P\right) \hat{z}+\mu z^{*}(t-\tau) D^{*} D z(t-\tau) \\
&+\hat{z}^{*}(I-\mu P)^{*} D z(t-\tau)+z^{*}(t-\tau) D^{*}(I-\mu P) \hat{z} .
\end{aligned}
$$

This completes the proof.

Lemma 7 Let $\omega>0, v>0$. If $(z, \hat{z})^{T}$ is a solution of (4), then $w=z^{*} z+\hat{z}^{*} \hat{z}$ satisfies

$$
\begin{aligned}
w^{\Delta} \leq & z^{*}\left(-A^{*}-A+\mu A^{*} A+\frac{1}{\omega}(I-\mu A)^{*}(I-\mu A)\right) z \\
& +(\mu+\omega) g^{*}(\hat{z}(t-\tau)) B^{*} B g(\hat{z}(t-\tau)) \\
& +\hat{z}^{*}\left(-P^{*}-P+\mu P^{*} P+\frac{1}{v}(I-\mu P)^{*}(I-\mu P)\right) \hat{z} \\
& +(\mu+v) z^{*}(t-\tau) D^{*} D z(t-\tau) .
\end{aligned}
$$

Proof First notice that for $\omega>0, v>0$ we have

$$
\begin{gathered}
0 \leq\left(\sqrt{\omega} B g(\hat{z}(t-\tau))-\frac{1}{\sqrt{\omega}}(I-\mu A) z\right)^{*} \\
\left(\sqrt{\omega} B g(\hat{z}(t-\tau))-\frac{1}{\sqrt{\omega}}(I-\mu A) z\right) \\
=\omega g^{*}(\hat{z}(t-\tau)) B^{*} B g(\hat{z}(t-\tau))-g^{*}(\hat{z}(t-\tau)) B^{*}(I-\mu A) z \\
-z^{*}(I-\mu A)^{*}(I-\mu A) g(\hat{z}(t-\tau)) \\
+\frac{1}{\omega} z^{*}(I-\mu A)^{*}(I-\mu A) z, \\
0 \leq\left(\sqrt{v} D z(t-\tau)-\frac{1}{\sqrt{v}}(I-\mu P) \hat{z}\right)^{*} \\
\left(\sqrt{v} D z(t-\tau)-\frac{1}{\sqrt{v}}(I-\mu P) \hat{z}\right) \\
=v z^{*}(t-\tau) D^{*} D z(t-\tau)-z^{*}(t-\tau) D^{*}(I-\mu P) \hat{z}
\end{gathered}
$$


$-\hat{z}^{*}(I-\mu P)^{*} D z(t-\tau)+\frac{1}{v} \hat{z}^{*}(I-\mu P)^{*}(I-\mu P) \hat{z}$.

Using this in Lemma 6, we can complete the proof.

Theorem 8 Suppose $g$ satisfies a Lipschitz condition

with Lipschitz constant $L$. Assume that

$A=\operatorname{diag}\left(a_{1}, a_{2}, \ldots, a_{n}\right), P=\operatorname{diag}\left(p_{1}, p_{2}, \ldots, p_{n}\right)$

are complex-valued diagonal matrix. If there exist

$\omega>0, v>0$ such that

$$
\phi:=\max \left\{\tilde{a}+(\mu+v) \lambda_{2}, \tilde{p}+(\mu+\omega) L^{2} \lambda_{1}\right\},
$$

satisfies $\phi \in \mathcal{R}^{+}$and $\lim _{t \rightarrow \infty} e_{\phi}\left(t, t_{0}\right)=0$, where $\lambda_{1}$ is the

maximal eigenvalue of $B^{*} B, \lambda_{2}$ is the maximal

eigenvalue of $D^{*} D$, and

$\tilde{a}:=\max _{1 \leq i \leq n}\left\{-2 \operatorname{Re}\left(a_{i}\right)+\mu\left|a_{i}\right|^{2}+\frac{1}{\omega}\left|1-\mu a_{i}\right|^{2}\right\}$,

$\tilde{p}:=\max _{1 \leq i \leq n}\left\{-2 \operatorname{Re}\left(p_{i}\right)+\mu\left|p_{i}\right|^{2}+\frac{1}{v}\left|1-\mu p_{i}\right|^{2}\right\}$,

then the trivial solution of (4) is $\phi$-globally

exponentially stable.

Proof Let $(z, \hat{z})^{T}$ be any solution of (4) and

let $w=w_{1}+w_{2}, w_{1}=z^{*} z, w_{2}=\hat{z}^{*} \hat{z}$. Then

$g^{*}(\hat{z}(t-\tau)) B^{*} B g(\hat{z}(t-\tau)) \leq L^{2} \lambda_{1} w_{2}$,

$z^{*}(t-\tau) D^{*} D z(t-\tau) \leq \lambda_{2} w_{1}$,

and

$z^{*}\left(-A^{*}-A+\mu A^{*} A+\frac{1}{\omega}(I-\mu A)^{*}(I-\mu A)\right) z \leq \tilde{a} w_{1}$,

$\hat{z}^{*}\left(-P^{*}-P+\mu P^{*} P+\frac{1}{v}(I-\mu P)^{*}(I-\mu P)\right) \hat{z} \leq \tilde{c} w_{2}$.

Thus ,by Lemma 7, we have

$w^{\Delta} \leq \tilde{a} w_{1}+(\mu+\omega) L^{2} \lambda_{1} w_{2}+\tilde{p} w_{2}+(\mu+v) \lambda_{2} w_{1} \leq \phi\left(w_{1}+w_{2}\right)=\phi w$.

Now Lemma 2 yields

$w(t) \leq w\left(t_{0}\right) e_{\phi}\left(t, t_{0}\right) \rightarrow 0 \quad$ as $\quad t \rightarrow \infty$,

so that the claim follows.

\section{Examples}

Consider the network

$\left\{\begin{array}{l}z^{\Delta}(t)=-\left(\begin{array}{cc}0.99 & 0 \\ 0 & 0.988\end{array}\right) z(t)+\left(\begin{array}{cc}0.025+0.025 i & -0.05+0.025 i \\ 0.075-0.05 i & -0.025+0.025 i\end{array}\right) g(\hat{z}(t-\tau)), \\ \hat{z}^{\Delta}(t)=-\left(\begin{array}{cc}0.99 & 0 \\ 0 & 0.995\end{array}\right) \hat{z}(t)+\left(\begin{array}{cc}0.035+0.035 i & -0.05+0.035 i \\ 0.065-0.05 i & -0.035+0.035 i\end{array}\right) z(t-\tau) .\end{array}\right.$

In (5), we choose the function

$$
g(x)=\left(\begin{array}{c}
\tanh \left(x_{1}+x_{2}\right) \\
\tanh \left(x_{1}+x_{2}\right)
\end{array}\right),
$$

the Lipschitz constant is equal to 1 and $\omega=v=1$, so that $\tilde{a}=-0.999712, \tilde{p}=-0.9998$. The two

eigenvalues of $B^{*} B$ can be computed as 0.0120 and

0.0017 . The two eigenvalues of $D^{*} D$ can be computed as 0.0017 and 0.0136 so that

$\lambda_{1}=0.0120, \lambda_{2}=0.0136$. Hence

$\phi=\max \{-0.999712+(\mu+\omega) 0.0136,-0.9998+(\mu+v) 0.0120\}$,

is constant if $\mu, \omega$ and $v$ are constant, in which case we

write $\alpha=\phi$.

1.For $T=R$ we have $\mu(t) \equiv 0$. We find

$\alpha=-0.986112$ and thus $e_{\alpha}(t, 0)=e^{\alpha t} \rightarrow 0$ as

$t \rightarrow \infty$ so that the trivial solution of (5) is $\alpha$-globally exponentially stable.

2.For $T=Z$ we have $\mu(t) \equiv 1$. We find $\alpha=-0.7598$ and thus $e_{\alpha}(t, 0)=(1+\alpha)^{t} \rightarrow 0$ as $t \rightarrow \infty$ so that the trivial solution of (5) is $\alpha$-globally exponentially stable.

\section{Discussion}

In this paper we have studied complex-valued genetic regulatory networks with delays on time scales. Sufficient conditions for the existence of a unique equilibrium solution are derived. The global exponential stability conditions derived in this paper are new, fairly general and offer greater flexibility in handing time scales of practical importance.

\section{Acknowledgement}

This research was supported by National Natural Science Foundation of China under Grant 61573005 and 11361010, the Foundation for Young Professors of Jimei University and the Foundation of Fujian Higher Education(JA11154,JA11144).

\section{References}

1. A. Hirose, Complex-Valued Neural Networks: Theories and Applications, World Scientific, Singapore, 2003.

2. M. Bohner, V. S. H. Rao, and S. Sanyal, Global stability of complex valued neural networks on time scales, Differ. Equ. Dyn. Syst., vol. 19, nos. 1 C 2, pp. 3 C 11, 2011.

3. V. S. H. Rao and G. R. Murthy, Global dynamics of a class of complex valued neural networks, Int. J. Neural Syst., vol. 18, no. 2, pp. 165 C 171,2008.

4. Y. Kuroe, N. Hashimoto, and T. Mori, On energy function for complex-valued neural networks and its applications, in Proc. 9th Int. Conf. Neural Inform. Process., Nov. 2002, pp. 1079 C 1083.

5. Y. Yao, Stability analysis for switched genetic regulatory networks: An average dwell time approach, Journal of the Franklin Institute, 348(2011) 2718 C 2733 